\title{
Impact of Baseline Characteristics on the Overall Survival of HCC Patients Treated with Sorafenib: Ten Years of Experience
}

\author{
Giulia Rovesti $^{a} \quad$ Giulia Orsi $^{\mathrm{a}}$ Andrikou Kalliopi ${ }^{\mathrm{a}}$ Caterina Vivaldi ${ }^{\mathrm{b}}$

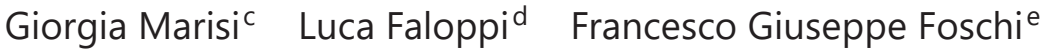 \\ Nicola Silvestris $^{f} \quad$ Irene Pecora $^{b}$ Giuseppe Aprile ${ }^{g}$ Eleonora Molinaro ${ }^{a}$ \\ Laura Riggi $^{a}$ Paola Ulivi ${ }^{c}$ Matteo Canale ${ }^{c}$ Alessandro Cucchetti ${ }^{h}$, \\ Emiliano Tamburini ${ }^{j}$ Giorgio Ercolani ${ }^{\mathrm{h}} \mathrm{h}$ Lorenzo Fornaro ${ }^{\mathrm{b}}$ \\ Pietro Andreone $^{k}$ Patrizia Zavattari $^{l}$ Mario Scartozzi $^{m}$ Stefano Cascinu ${ }^{n}$ \\ Andrea Casadei-Gardini ${ }^{a}$ \\ a Division of Oncology, Department of Oncology and Hematology, University Hospital of \\ Modena, Modena, Italy; ${ }^{b}$ Department of Medical Oncology, Pisa University Hospital, Pisa, \\ Italy; ' ${ }^{\mathrm{B}}$ Biosciences Laboratory, Istituto Scientifico Romagnolo per lo Studio e la Cura dei \\ Tumori (IRST) IRCCS, Meldola, Italy; 'Oncology Unit, Macerata Hospital, Macerata, Italy; \\ e Department of Internal Medicine, Faenza Hospital, Faenza, Italy; ${ }^{f}$ IRCCS Istituto Tumori \\ "Giovanni Paolo II", Bari, Italy; ${ }^{9}$ Medical Oncology Unit, Hospital of Vicenza, Vicenza, Italy; \\ ${ }^{h}$ Department of Medical and Surgical Sciences-DIMEC, S. Orsola-Malpighi Hospital, Alma

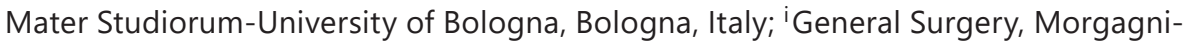 \\ Pierantoni Hospital, Forlì, Italy; jDepartment of Oncology, Ospedale Cardinale G. Panico, \\ Tricase, Italy; ${ }^{k}$ Division of Internal and Metabolic Medicine, Baggiovara Hospital, University \\ of Modena and Reggio Emilia, Modena, Italy; 'Unit of Biology and Genetics, Department of \\ Biomedical Sciences, University of Cagliari, Cagliari, Italy; ${ }^{m}$ Department of Medical Sciences \\ and Public Health, University of Cagliari, Cagliari, Italy; ${ }^{n}$ Department of Medical Oncology, \\ Università Vita-Salute, San Raffaele Hospital IRCCS, Milan, Italy
}

\section{Keywords}

Real-life conditions · Baseline factors · ALBI grade - Etiologies · Child-Pugh · ECOG ·

Neutrophil-to-lymphocyte ratio $\cdot$ Lactate dehydrogenase $\cdot$ Prognostic factor - Transaminases

\section{Abstract}

Background: Sorafenib has been established as the standard of care for patients with advanced hepatocellular carcinoma (HCC) since 2007 on the basis of two landmark trials (SHARP and Asia-Pacific). Ten years have passed since then and, despite much research in the field,

Mario Scartozzi, Stefano Cascinu, and Andrea Casadei Gardini are last co-authors. 
still no validated real-life prognostic markers are available for HCC patients treated with this drug. Therefore, going through 10 years of research into sorafenib of several Italian Cancer Centers, we conducted a field-practice study aimed at identifying baseline clinical factors that could be significantly associated with overall survival (OS). Method: Univariate/multivariate analyses were conducted to retrospectively identify the impact of baseline characteristics on the OS of 398 advanced HCC patients treated with sorafenib. Results: Based on univariate analysis, $\alpha$-fetoprotein (AFP), albumin, AST, bilirubin, Child-Pugh, ECOG, systemic immuneinflammation index (SII), albumin-bilirubin (ALBI) grade, and portal vein thrombosis were significantly associated with shorter OS. Following adjustment for clinical covariates positive in univariate analysis, the multivariate analysis including AFP, age, etiology, albumin, aspartate transaminase (AST), bilirubin, Child-Pugh, LDH, platelet-to-lymphocyte ratio, ECOG, ALBI grade, portal vein thrombosis, SII, and BCLC stage identified increase in $\mathrm{LDH}$, age $>70$ years, no viral etiologies, ECOG $>0$, albumin $<35$, ALBI grade 2, and AST $>40$ as prognostic factors for poorer OS based on the $5 \%$ significance level. Conclusion: Our study highlights that baseline hepatic function, patient-centered variables, and etiology have prognostic value. These findings might have implications in terms of therapeutic decision-making and patient counseling.

(C) 2019 S. Karger AG, Basel

\section{Introduction}

Hepatocellular carcinoma (HCC), the most frequent primary liver malignancy, ranks as the sixth cancer worldwide in terms of incidence and represents the second leading cause of cancer-related death [1-3]. A peculiarity of this neoplasm is its tendency to develop in the context of an advanced liver disease in the vast majority of cases. Etiologies vary from wellknown predisposing conditions such as chronic viral infections (HCV and HBV) and alcohol abuse, to more recent and increasing risk factors which are part of the spectrum of the metabolic diseases (non-alcoholic steatohepatitis [NASH], non-alcoholic fatty liver disease, obesity, diabetes) $[4,5]$.

Sorafenib has represented the cornerstone of HCC systemic treatment since 2007 and the only FDA-approved agent for frontline therapy of advanced HCC until 2018. In the phase III SHARP trial, sorafenib demonstrated a significant improvement in overall survival (OS) in comparison to placebo for the treatment of advanced HCC (10.7 vs. 7.9 months; hazard ratio [HR] 0.69, 95\% CI: 0.55-0.87; $p<0.001$ ) [6]. The most frequent adverse events (AEs) were hand-foot syndrome and diarrhea, with a manageable toxicity profile [6]. These data were subsequently confirmed in another phase III, placebo-controlled trial involving HCC patients of the Asia-Pacific area, with an OS benefit of 2.3 months reported in the sorafenib arm [7]. The role of sorafenib for HCC treatment under real-life practice conditions has been further assessed in the GIDEON trial (Global Investigation of therapeutic DEcisions in hepatocellular carcinoma and of its treatment with sorafeNib) [8]. This observational study involving more than 3,200 unresectable HCC patients, showed that sorafenib tolerability was not significantly different between Child-Pugh (C-P) A and B subgroups [8], but C-P B patients exhibited poorer survival outcomes compared to C-P A group [8]. In this study, no analysis on prognostic or predictive factors to sorafenib was performed.

Among the new available agents for HCC treatment, lenvatinib, an oral inhibitor of multiple receptor tyrosine kinases, received FDA approval as first-line therapy for advanced HCC in 2018, after its non-inferiority in comparison to sorafenib was reported in the REFLECT trial [9]. In September 2017, the FDA granted accelerated approval to the fully human IgG4 
monoclonal antibody programmed death receptor-1 (PD-1) inhibitor nivolumab for the treatment of HCC patients previously treated with sorafenib, based on the results of the phase I/II CheckMate-040 trial [10]. Now, great expectation is addressed towards the ongoing phase III CheckMate-459 trial (NCT02576509), which compares nivolumab and sorafenib as firstline therapy for advanced-stage HCC patients [11].

Despite this evolving scenario, the role of sorafenib remains pivotal, also because of more than 10 years' experience gained in the management of its activity and safety profile [4]. Unfortunately, the main results obtained to date come from 2 important randomized trials and from different subanalyses and pooled analyses, rather than from normal clinical practice. With that in mind, exploring the characteristics and the possible prognostic factors in a population of HCC patients treated with sorafenib under real-life conditions in the last 10 years becomes of paramount importance.

After 10 years of research into sorafenib, there are still no validated prognostic or predictive factors of response to the drug in HCC [12]. The aim of the present study was to summarize 10 years of research into sorafenib in the real-life experience of several Italian Cancer Centers, looking for clinical factors that could be significantly associated with survival outcomes in this setting of patients.

\section{Patients and Methods}

\section{Patients and Treatment}

This multicenter Italian study was conducted on HCC patients consecutively treated and derived from our retrospective studies conducted in these years [13-22]. Patients receiving sorafenib with advancedstage and intermediate-stage HCC refractory to or unsuitable for locoregional therapies, either histologically proven or diagnosed according to the AASLD (American Association for the Study of Liver Diseases 2005) guidelines, were eligible for our analysis. None of the patients had received previous systemic therapy. Eligibility criteria were the same as those of Llovet's pivotal study on sorafenib in HCC [6].

All patients received sorafenib according to standard schedule (400 mg b.i.d. continuously) or physician choice; dose reduction was applied as clinically indicated. Follow-up consisted of CT/MRI scan every 8 weeks or as clinically indicated. Tumor response was evaluated by modified Response Evaluation Criteria in Solid Tumors (mRECIST). Treatment with sorafenib was continued until disease progression, unacceptable toxicity, or death.

\section{Statistical Analysis}

The aim of this analysis was to examine the association between baseline characteristics and OS in patients with HCC treated with sorafenib. All hematologic blood tests were carried out at baseline the day before the start of the treatment. Association between categorical variables was assessed using Fisher's exact test, when appropriate.

OS was defined as the time interval between the time from the start date of treatment to the date of death or last follow-up visit. OS were estimated by the Kaplan-Meier method and curves were compared by the log-rank test.

The multivariate analyses were conducted using seven models. Model 1 included all basal positive analysis that we found in univariate analysis plus age and gender. In the other models, we added in various steps all other analyses (model 2: model 1 plus previous treatment; model 3: model 1 plus response to sorafenib; model 4: model 1 plus starting dosage; model 5: model 1 plus treatment in second line; model 6: model 1 plus skin toxicity; model 7: model 1 plus all toxicity).

Backward selection was used with a threshold level of $10 \%$ to select variables to evaluate in the MV analysis. The significance level of $5 \%$ was used to determine statistical significance. MedCalc package (MedCalc ${ }^{\circledR}$ version 16.8.4) was used for all statistical analysis. 


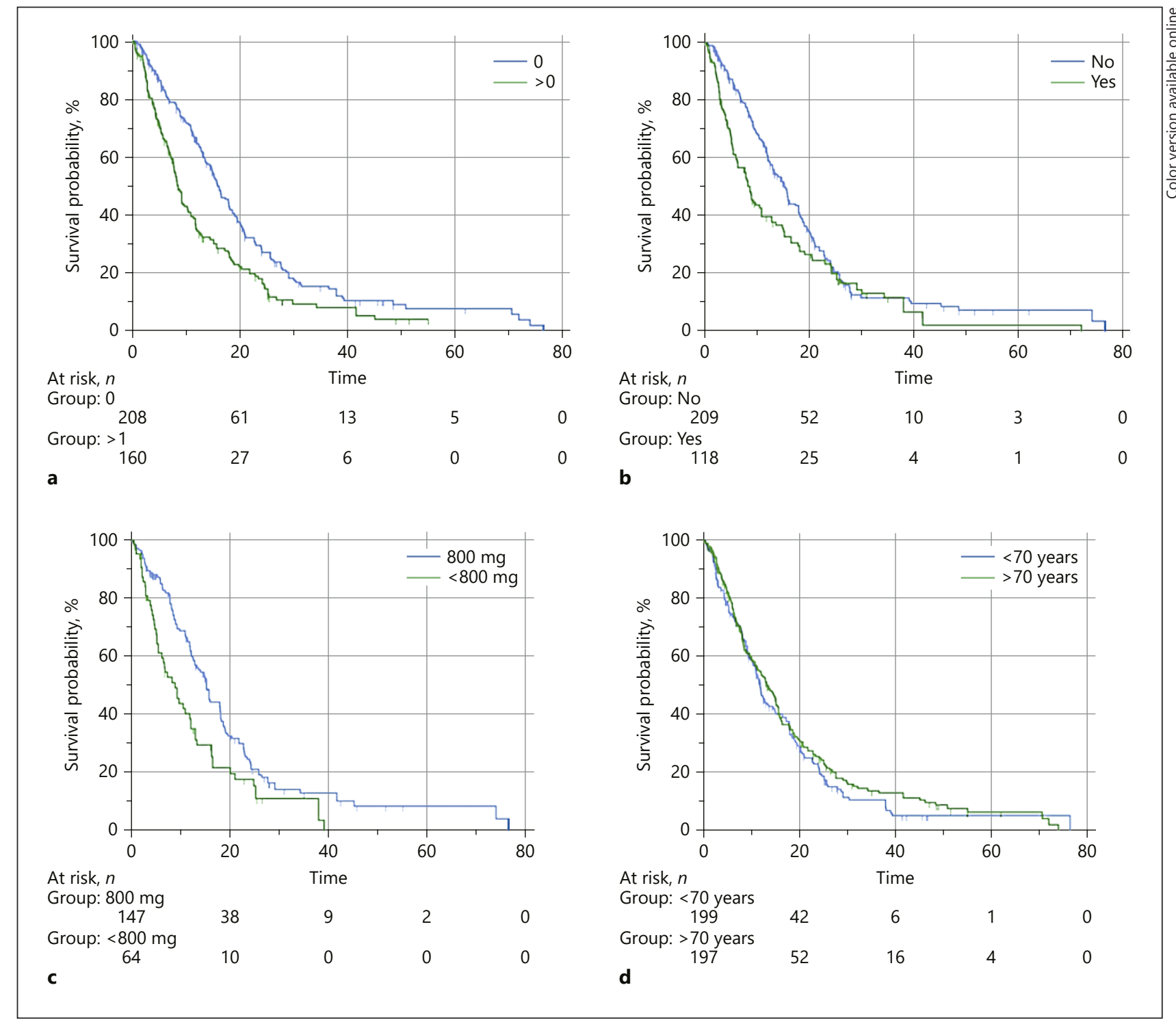

Fig. 1. Overall survival in relation to ECOG (a), portal vein thrombosis (b), starting dosage (c), and age (d).

\section{Results}

Between June 7th, 2007, and August 8th, 2018, 398 patients with HCC and treated with sorafenib were included in our analysis. The study sample included 356 males (89.4\%) and 42 females $(10.6 \%)$ with a median age at diagnosis of 69 years (range 25-88). Median OS was 12 months (95\% CI: 11.1-14.6). Median follow-up was 36 months (range 1-65).

C-P class A was the most represented ( $n=255 ; 88.1 \%) .263$ patients $(71.2 \%)$ had BCLC-C disease stage. $28.2 \%$ patients had an $\alpha$-fetoprotein (AFP) level $>400 \mathrm{ng} / \mathrm{mL}$. The most common underlying etiology was hepatitis infection from $C$ virus $(52.6 \%)$ and $B$ virus $(20.1 \%)$.

Sorafenib was started at the standard dose of $800 \mathrm{mg} /$ day in $69.6 \%$ of patients. AEs of any grade were reported in 273 patients (74.5\%); 32.2\% of patients reported hand-foot skin toxicity (grade 1: 27.9\%; grade 2: 50\%; grade 3: 20.3\%). Best response to sorafenib treatment was represented by 2 complete responses $(0.7 \%)$, partial response in 36 patients $(11.5 \%)$, 
Table 1. Univariate analysis of basal characteristics

\begin{tabular}{|c|c|c|c|c|c|c|}
\hline & $\begin{array}{l}\text { Patients, } \\
n\end{array}$ & $\begin{array}{l}\text { Events, } \\
n\end{array}$ & $\begin{array}{l}\text { Median OS, } \\
\text { months (95\% CI) }\end{array}$ & HR & $95 \% \mathrm{CI}$ & $p$ value \\
\hline Age at diagnosis (c.v.) & 396 & 314 & & 1.00 & $0.98-1.01$ & 0.4683 \\
\hline \multicolumn{7}{|l|}{ Age at diagnosis } \\
\hline$<80$ years & 355 & 281 & 12.3 (11.1-14.9) & 1 & & \\
\hline$>80$ years & 41 & 33 & $11.7(6.1-17.8)$ & 1.29 & $0.86-1.91$ & 0.2136 \\
\hline \multicolumn{7}{|l|}{ Sex } \\
\hline $1(\mathrm{M})$ & 356 & 281 & $12.4(10.8-14.9)$ & 1 & & \\
\hline $2(\mathrm{~F})$ & 42 & 35 & $12.3(11.0-16.1)$ & 1.01 & $0.71-1.44$ & 0.9459 \\
\hline \multicolumn{7}{|l|}{ BCLC stage } \\
\hline B & 106 & 80 & $15.6(12.4-19.0)$ & 1 & & \\
\hline $\mathrm{C}$ & 263 & 209 & $11.8(9.2-13.5)$ & 1.20 & $0.94-1.55$ & 0.1382 \\
\hline \multicolumn{7}{|l|}{ Etiology } \\
\hline Alcohol & 37 & 30 & $13.9(7.9-20.8)$ & 1 & & 0.5594 \\
\hline Others & 39 & 32 & $12.0(9.2-15.1)$ & 1.34 & $0.80-2.26$ & \\
\hline HBV & 79 & 65 & $11.3(8.8-15.0)$ & 1.18 & $0.76-1.81$ & \\
\hline $\mathrm{HCV}$ & 207 & 161 & $13.5(11.1-15.8)$ & 1.01 & $0.69-1.48$ & \\
\hline NASH & 31 & 24 & $12.9(6.1-15.6)$ & 1.02 & $0.60-1.72$ & \\
\hline Body mass index (c.v.) & 237 & 180 & & 0.99 & $0.95-1.03$ & 0.7956 \\
\hline \multicolumn{7}{|l|}{ Body mass index } \\
\hline Low & 8 & 6 & $10.8(7.7-26.3)$ & 1.23 & $0.50-3.01$ & 0.8845 \\
\hline Normal & 208 & 157 & $13.2(12.0-15.6)$ & 1 & & \\
\hline High & 21 & 17 & $11.8(8.0-22.8)$ & 1.00 & $0.61-1.66$ & \\
\hline \multicolumn{7}{|l|}{ Performance status } \\
\hline 0 & 208 & 158 & $15.8(14.3-18.0)$ & 1 & & $<0.0001$ \\
\hline$>0$ & 160 & 131 & $8.5(7.7-10.3)$ & 1.72 & $1.34-2.20$ & \\
\hline \multicolumn{7}{|l|}{ Portal vein thrombosis } \\
\hline No & 209 & 151 & $15.1(12.9-17.8)$ & 1 & & 0.0065 \\
\hline Yes & 118 & 99 & $8.5(6.1-10.8)$ & 1.45 & $1.11-1.90$ & \\
\hline \multicolumn{7}{|c|}{ Sorafenib dosage (800 vs. other) } \\
\hline 800 & 147 & 112 & $15.1(12.7-17.9)$ & 1 & & 0.0015 \\
\hline Other & 64 & 54 & 8.7 (5.3-12.0) & 1.82 & $1.26-2.63$ & \\
\hline
\end{tabular}

stable disease in 145 patients (46.5\%), and progression disease in 129 patients (41.3\%); the disease control rate was $58.5 \% .44 .5 \%$ of patients received other treatments after sorafenib progression/intolerance.

\section{Basal Characteristics and Clinical Outcome}

For basal characteristics, the univariate analysis identified ECOG $>0$ (vs. 0 ; HR 1.72; $p<$ 0.0001 ) (Fig. 1a), portal vein thrombosis yes (vs. no; HR 1.45; $p=0.0065$ ) (Fig. 1b) and starting dosage $<800 \mathrm{mg}$ (vs. $800 \mathrm{mg}$; HR 1.82; $p=0.0015$ ) (Fig. 1c) as potential prognostic factors for poorer OS (Fig. 1d). At univariate analysis, no differences were found for age (Fig. 1d), gender, etiology and body mass index (Table 1).

For hepatic function, the univariate analysis identified C-P B (vs. A; HR 1.57; $p=0.0220$ ) (Fig. 2a), albumin $<35$ (vs. $>35$; HR 1.47; $p=0.0317$ ) (Fig. 2b), bilirubin $>$ normal value (NV) (vs. $<$ NV; HR 1.43; $p=0.0297$ ) (Fig. 2c), albumin-bilirubin (ALBI) grade 2 (vs. 1; HR 1.76; $p=$ 
Gastro

Intestinal

Tumors
Gastrointest Tumors 2019;6:92-107

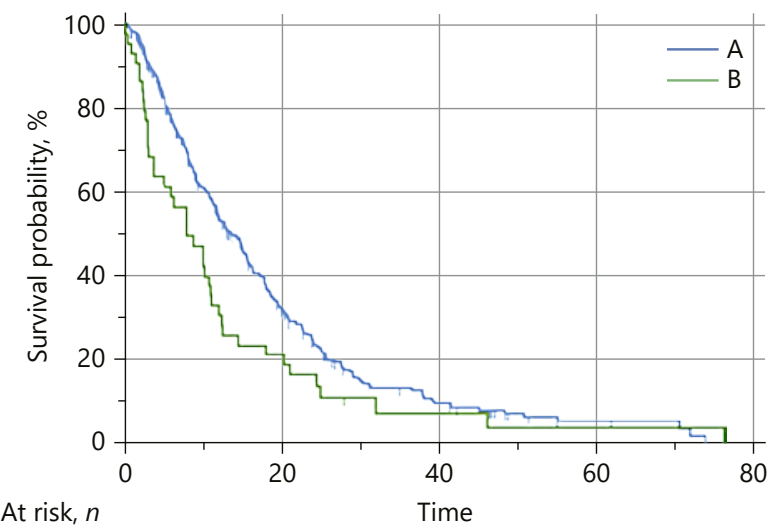

At risk, $n$

Group: A

Group: B 327

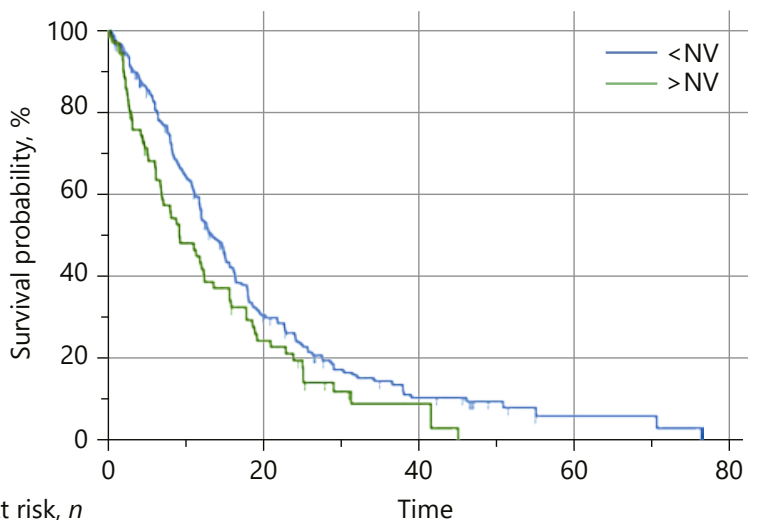

At risk, $n$

Group: $<$ NV 193

Group: $>$ NV

c

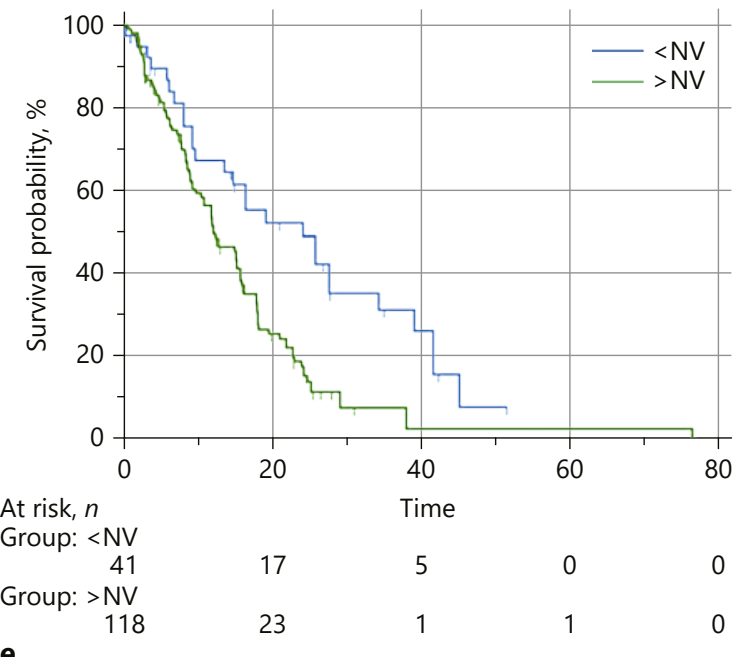

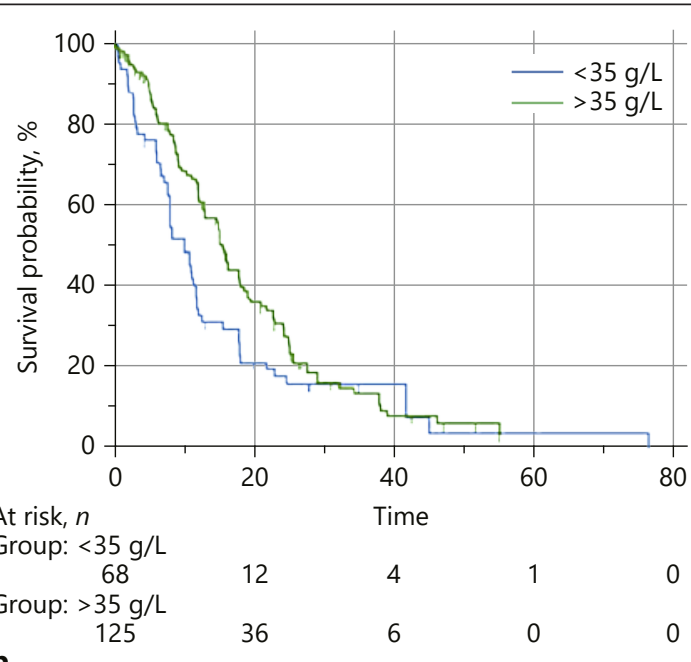
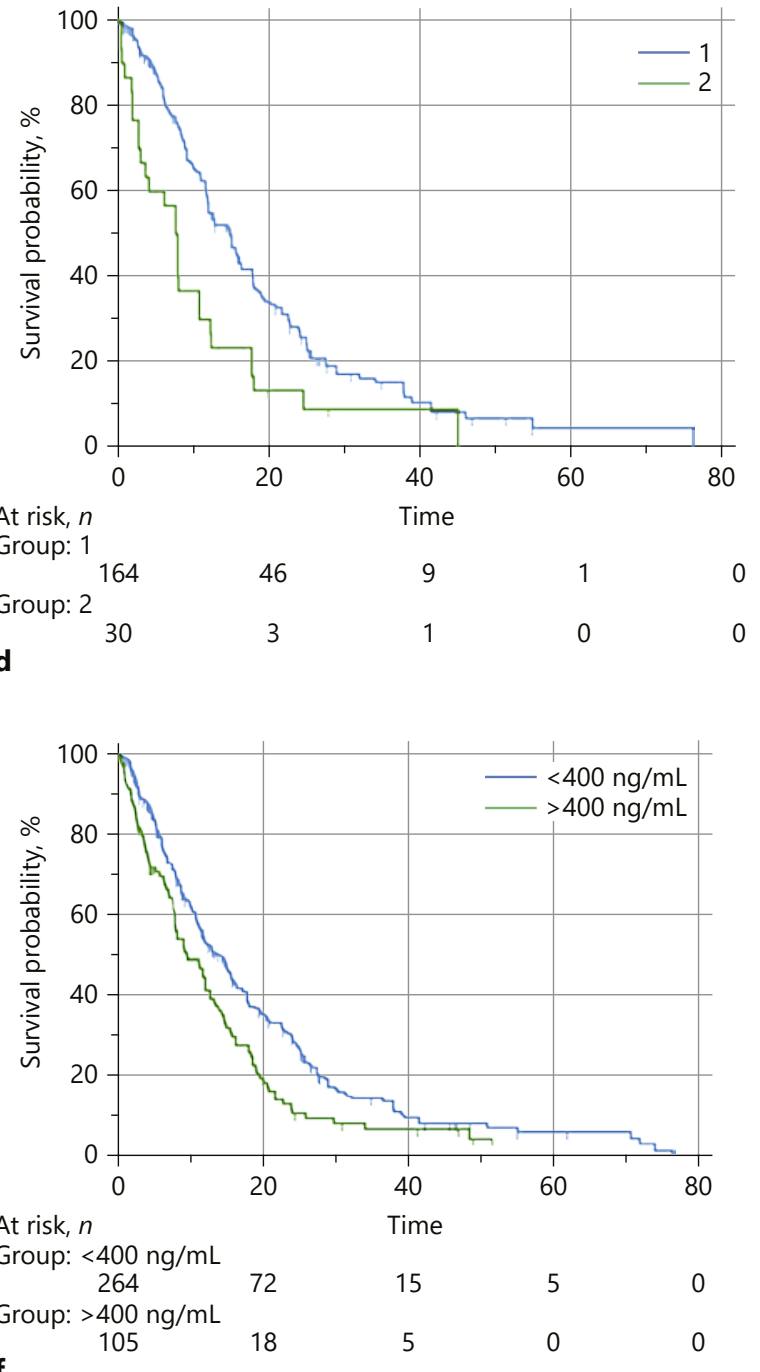

Fig. 2. Overall survival in relation to Child-Pugh (a), albumin (b), bilirubin (c), ALBI (d), AST (e), and $\alpha$-fetoprotein (f). 
Tumors

Table 2. Univariate analysis of liver function

\begin{tabular}{|c|c|c|c|c|c|c|}
\hline & $\begin{array}{l}\text { Patients, } \\
n\end{array}$ & $\begin{array}{l}\text { Events, } \\
n\end{array}$ & $\begin{array}{l}\text { Median OS, } \\
\text { months }(95 \% \mathrm{CI})\end{array}$ & HR & $95 \% \mathrm{CI}$ & $p$ value \\
\hline \multicolumn{7}{|l|}{ Child-Pugh } \\
\hline A & 327 & 255 & $13.5(11.8-15.6)$ & 1 & & 0.0220 \\
\hline B & 44 & 41 & $8.0(3.7-10.9)$ & 1.57 & $1.07-2.33$ & \\
\hline Albumin (c.v.) & 198 & 157 & & 0.94 & $0.91-0.98$ & 0.0066 \\
\hline \multicolumn{7}{|l|}{ Albumin } \\
\hline$<35$ & 68 & 58 & 10.0 (7.9-11.7) & 1.47 & $1.03-2.09$ & 0.0317 \\
\hline$>35$ & 125 & 94 & $15.6(12.7-18.0)$ & 1 & & \\
\hline Bilirubin (c.v.) & 262 & 215 & & 1.32 & $1.02-1.71$ & 0.0317 \\
\hline \multicolumn{7}{|l|}{ Bilirubin } \\
\hline$<1.2$ & 193 & 155 & $13.5(11.8-15.6)$ & 1 & & 0.0297 \\
\hline$>1.2$ & 71 & 61 & $9.2(6.8-13.6)$ & 1.43 & $1.03-1.98$ & \\
\hline ALBI grade (c.v.) & 194 & 155 & & 1.76 & $1.19-2.60$ & 0.0040 \\
\hline \multicolumn{7}{|l|}{ ALBI grade } \\
\hline 1 & 164 & 127 & $14.9(12.0-16.3)$ & 1 & & 0.0008 \\
\hline 2 & 30 & 28 & $7.7(3.0-10.8)$ & 2.47 & $1.46-4.20$ & \\
\hline ALT (c.v.) & 190 & 148 & & 1.00 & $0.99-1.00$ & 0.1433 \\
\hline \multicolumn{7}{|l|}{ ALT } \\
\hline$<41$ & 79 & 58 & $13.5(9.6-18.5)$ & 1 & & 0.4356 \\
\hline$>41$ & 107 & 86 & $12.9(10.8-15.8)$ & 1.14 & $0.81-1.60$ & \\
\hline AST (c.v.) & 162 & 124 & & 1.00 & $1.00-1.00$ & 0.0094 \\
\hline \multicolumn{7}{|l|}{ AST } \\
\hline$<40$ & 41 & 27 & $24.0(13.5-34.1)$ & 1 & & 0.0010 \\
\hline$>40$ & 118 & 94 & $12.0(10.4-15.6)$ & 1.92 & $1.30-2.85$ & \\
\hline \multicolumn{7}{|l|}{ Ascites } \\
\hline No & 59 & 45 & $13.9(10.4-17.8)$ & 1 & & 0.0045 \\
\hline Yes & 35 & 30 & $4.1(2.2-9.7)$ & 2.16 & $1.27-3.68$ & \\
\hline Alkaline phosphatase (c.v) & 59 & 43 & & 1.00 & $0.99-1.00$ & 0.3421 \\
\hline \multicolumn{7}{|l|}{ Alkaline phosphatase } \\
\hline$<129$ & 23 & 17 & $14.6(8.7-20.9)$ & 1 & & 0.6684 \\
\hline$>129$ & 36 & 26 & $9.6(3.1-17.8)$ & 1.14 & $0.61-2.11$ & \\
\hline GGT (c.v.) & 61 & 44 & & 1.00 & $0.99-1.00$ & 0.7878 \\
\hline \multicolumn{7}{|l|}{ GGT } \\
\hline$<61$ & 4 & 4 & $5.8(2.2-14-6)$ & 2.55 & & 0.1877 \\
\hline$>61$ & 57 & 40 & $13.5(5.6-17.8)$ & 1 & $0.63-10.25$ & \\
\hline MELD (c.v.) & 347 & 272 & & 1.04 & $0.99-1.08$ & 0.1132 \\
\hline \multicolumn{7}{|l|}{ MELD } \\
\hline$<12$ & 295 & 224 & $12.8(11.3-15.0)$ & 1 & & 0.5322 \\
\hline$>12$ & 52 & 48 & $10.9(6.8-17.8)$ & 1.11 & $0.80-1.54$ & \\
\hline
\end{tabular}


Table 3. Univariate analysis of other basal blood tests

\begin{tabular}{|c|c|c|c|c|c|c|}
\hline & $\begin{array}{l}\text { Patients, } \\
n\end{array}$ & $\begin{array}{l}\text { Events, } \\
n\end{array}$ & $\begin{array}{l}\text { Median OS, } \\
\text { months }(95 \% \mathrm{CI})\end{array}$ & $\mathrm{HR}$ & $95 \% \mathrm{CI}$ & $p$ value \\
\hline AFP (c.v.) & 372 & 296 & & 1.00 & $1.00-1.00$ & 0.9032 \\
\hline \multicolumn{7}{|l|}{ AFP } \\
\hline$<400$ & 264 & 202 & $14.3(11.4-16.1)$ & 1 & & 0.0028 \\
\hline$>400$ & 105 & 91 & $9.6(7.9-12.7)$ & 1.51 & $1.15-1.98$ & \\
\hline Serum calcium (c.v.) & 155 & 123 & & 0.84 & $0.61-1.16$ & 0.2994 \\
\hline \multicolumn{7}{|l|}{ Serum calcium } \\
\hline$<8.6$ & 14 & 12 & $11.2(4.2-16.3)$ & 1.45 & $0.73-2.88$ & \\
\hline$>8.6$ & 131 & 102 & $12.9(10.9-15.6)$ & 1 & & 0.2907 \\
\hline Creatinine (c.v.) & 267 & 219 & & 1.11 & $0.69-1.79$ & 0.6612 \\
\hline \multicolumn{7}{|l|}{ Creatinine } \\
\hline$<1.2$ & 237 & 190 & $12.0(11.3-14.9)$ & 1.02 & & 0.9111 \\
\hline$>1.2$ & 30 & 29 & $11.1(6.7-22.8)$ & 1 & $0.69-1.52$ & \\
\hline $\mathrm{Hb}$ (c.v.) & 221 & 176 & & 0.98 & $0.90-1.06$ & 0.5887 \\
\hline \multicolumn{7}{|l|}{$\mathrm{Hb}$} \\
\hline$<\mathrm{NV}$ & 111 & 91 & $11.2(8.5-12.7)$ & 1.10 & $0.81-1.48$ & 0.5330 \\
\hline NV & 110 & 85 & $15.6(12.0-18.0)$ & 1 & & \\
\hline INR (c.v.) & 209 & 168 & & 1.19 & $0.83-1.70$ & 0.3444 \\
\hline LDH (c.v.) & 174 & 143 & & 1.00 & $1.00-1.00$ & 0.0210 \\
\hline \multicolumn{7}{|l|}{ LDH } \\
\hline$<225$ & 63 & 48 & $14.7(10.4-18.0)$ & 1 & & 0.6538 \\
\hline$>225$ & 111 & 95 & $12.0(8.5-15.1)$ & 1.08 & $0.76-1.53$ & \\
\hline Serum sodium (c.v.) & 196 & 160 & & 0.96 & $0.93-1.00$ & 0.0524 \\
\hline \multicolumn{7}{|l|}{ Serum sodium } \\
\hline$<136$ & 41 & 33 & $9.2(6.0-16.1)$ & 1.36 & $0.89-2.07$ & 0.1559 \\
\hline$>136$ & 155 & 127 & $12.0(11.0-15.1)$ & 1 & & \\
\hline
\end{tabular}

0.004) (Fig. 2d), and aspartate transaminase (AST) $>$ NV (vs. $<$ NV; HR 1.92; $p=0.001$ ) (Fig. 2e) as potential prognostic factors for poorer OS. No differences were found for alanine transaminase (ALT), alkaline phosphatase, GGT (gamma glutamyl transferase), Meld score, and BCLC stage (Table 2). For other parameters, only AFP $>400$ (vs. $<400$; HR 1.51; $p=0.0028$ ) (Fig. 2f) and LDH as continuous variable (HR 1.00; $p=0.021$ ) were found as potential prognostics factors (Table 3).

For inflammatory index, the univariate analysis identified platelet-to-lymphocyte ratio (PLR) as continuous variable (HR 4.94; $p=0.026$ ) and systemic immune-inflammation index (SII) $>360$ (vs. $<360$; HR 1.36; $p=0.041$ ) as potential prognostic factor for poorer OS. Conversely, neutrophil-to-lymphocyte ratio (NLR) (cut off of 3 ) was not associated with a different clinical outcome.

Other Parameters and Clinical Outcome

Regarding previous treatments, the univariate analysis identified previous treatment with radiofrequency (RFA) (vs. no RFA; HR 1.67; $p=0.0017$ ) (Fig. 3a) and transarterial chemoembolization (TACE) (vs. no TACE; HR 1.40; $p=0.0060$ ) (Fig. 3b) as potential prog- 
Gastro

Intestinal

Tumors
Gastrointest Tumors 2019:6:92-107

Rovesti et al.: Main Factors in Sorafenib Outcome

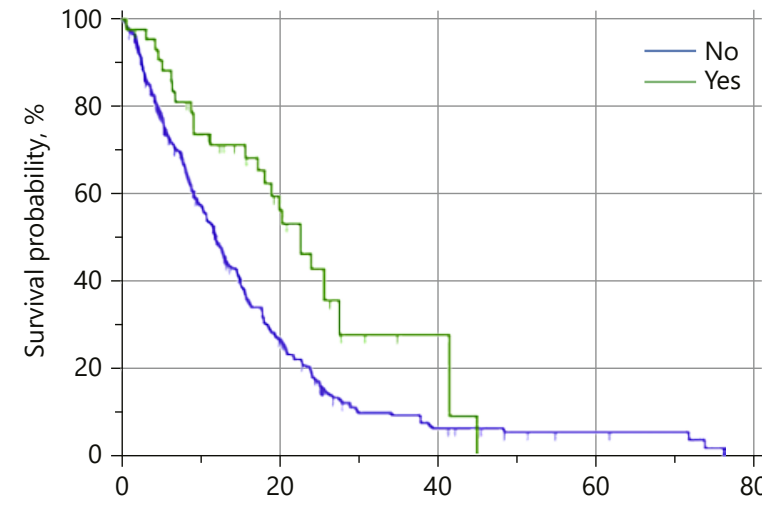

At risk, $n$

Time

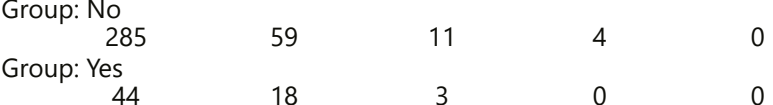

a

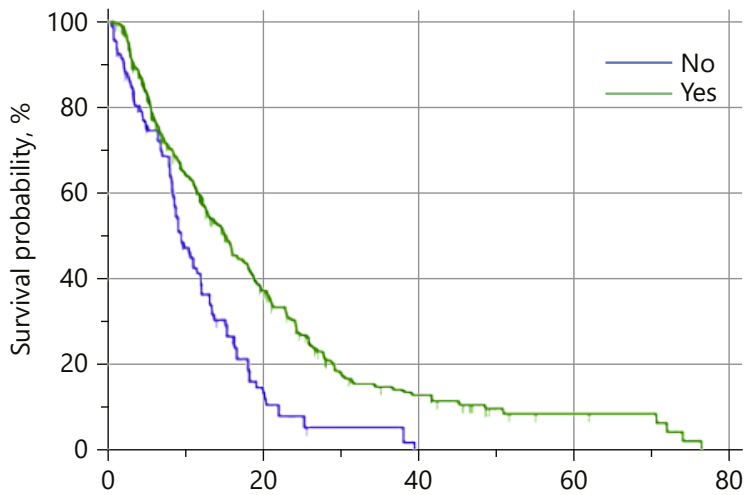

At risk, $n$

Time

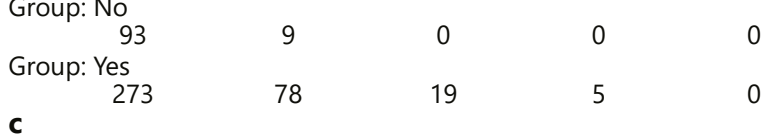

C

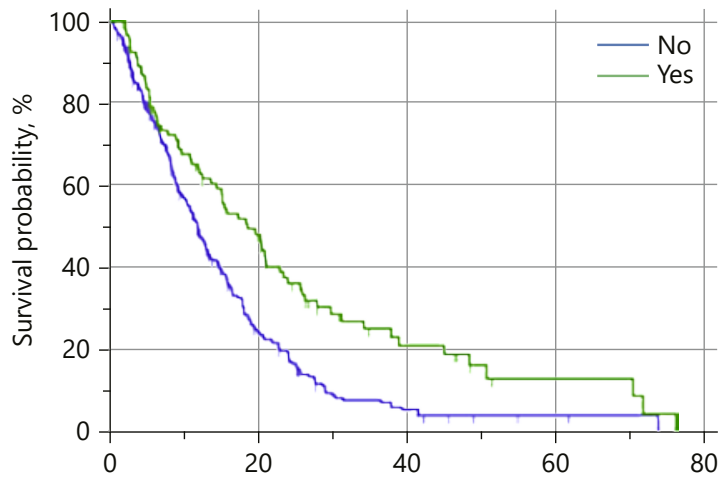

At risk, $n$

Time

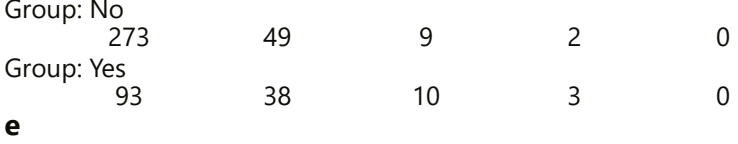

Fig. 3. Overall survival in relation to previous treatment with RFA (a), TACE (b), all toxicity (c), hand-foot skin toxicity (d), diarrhea (e), and second-line treatment (f).
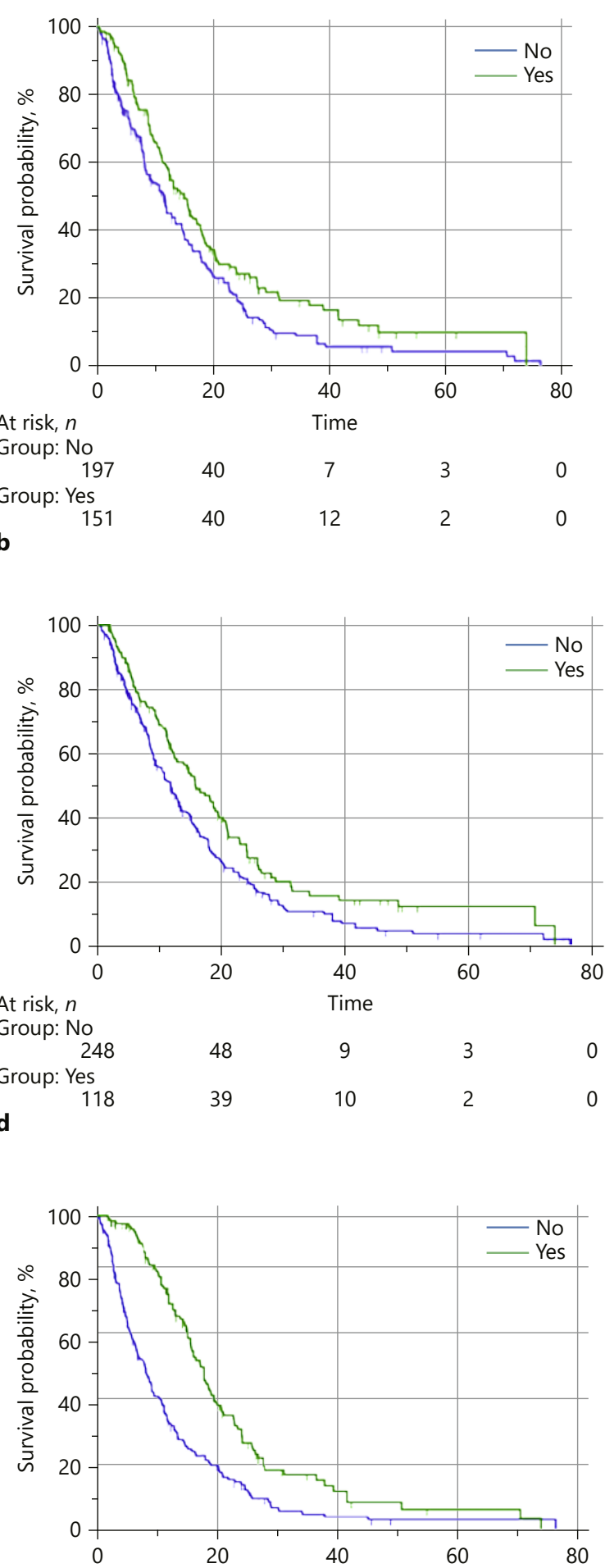

At risk, $n$

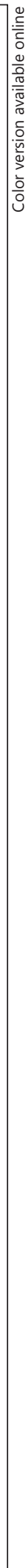


Table 4. Multivariate analysis

\begin{tabular}{|c|c|c|c|c|c|c|c|}
\hline $\mathrm{AFP}>400$ & 0.1335 & 0.3171 & 0.8571 & 0.0957 & 0.4283 & 0.1833 & 0.2667 \\
\hline LDH & 0.0414 & 0.0742 & 0.0383 & 0.0480 & 0.0613 & 0.0463 & 0.0689 \\
\hline Age $<70$ & 0.0007 & 0.0001 & 0.0414 & 0.0039 & 0.0018 & 0.0004 & 0.0005 \\
\hline \multicolumn{7}{|l|}{ Etiology } & \\
\hline HBV & 0.2004 & 0.0656 & 0.0832 & 0.2638 & 0.7268 & 0.0886 & 0.1658 \\
\hline Others & 0.0038 & 0.0198 & 0.0031 & 0.0039 & 0.0028 & 0.0023 & 0.0014 \\
\hline $\mathrm{PS}>1$ & 0.0305 & 0.2400 & 0.0579 & 0.0302 & 0.0331 & 0.0347 & 0.0548 \\
\hline Portal vein thrombosis & 0.5814 & 0.9398 & 0.8212 & 0.6105 & 0.2057 & 0.5893 & 0.7544 \\
\hline PLR $>16$ & 0.4666 & 0.5075 & 0.7603 & 0.3811 & 0.9062 & 0.6422 & 0.5831 \\
\hline SII $<360$ & 0.3499 & 0.2440 & 0.5673 & 0.4088 & 0.2896 & 0.3068 & 0.4079 \\
\hline Child-Pugh B & 0.1304 & 0.2885 & 0.0030 & 0.1181 & 0.1940 & 0.1466 & 0.1688 \\
\hline Albumin $<35$ & 0.0004 & 0.0018 & 0.0987 & 0.0013 & 0.0021 & 0.0007 & 0.0007 \\
\hline Bilirubin $>1.2$ & 0.6055 & 0.2961 & 0.7319 & 0.7118 & 0.5484 & 0.9362 & 0.6926 \\
\hline ALBI 2 & $<0.0001$ & $<0.0001$ & 0.0001 & $<0.0001$ & $<0.0001$ & $<0.0001$ & $<0.0001$ \\
\hline AST $<40$ & 0.0074 & 0.0885 & 0.0023 & 0.0131 & 0.0567 & 0.0027 & 0.0101 \\
\hline BCLC B & 0.8875 & 0.4047 & 0.6065 & 0.8442 & 0.9231 & 0.7806 & 0.9488 \\
\hline Thermoablation & & 0.0077 & & & & & \\
\hline TACE & & 0.7452 & & & & & \\
\hline Response PD & & & 0.0131 & & & & \\
\hline Dosage $<800 \mathrm{mg}$ & & & & 0.1327 & & & \\
\hline Subsequent treatments & & & & & 0.1375 & & \\
\hline Skin toxicity & & & & & & 0.6407 & \\
\hline Any toxicity & & & & & & & 0.1553 \\
\hline
\end{tabular}

nostic factors for greatest OS. The number of TACE and the best response were not associated with different clinical outcome.

For toxicity, we found a correlation between toxicity (all toxicity [Fig. 3c], hand-foot skin toxicity [Fig. 3d], and diarrhea [Fig. 3e]) and good prognosis. No second-line treatment was associated with a lower survival (HR 2.07; $p<0.0001$ ) (Fig 3f).

Regarding best response to sorafenib, we found a correlation between them and clinical outcome (complete response HR 1.00; partial response HR 1.61; stable disease HR 2.75; progression disease HR 7.51; $p<0.0001$ ). Finally, we analyzed six categories of concomitant drug, but no correlation between them and clinical outcome was found.

\section{Multivariate Analysis}

The multivariate analysis using model 1 (including AFP [cut off 400], age [ $<$ or $\geq 70$ years], etiology [HBV, HCV and other], albumin [< or $>$ NV], AST $[<$ or $>$ NV], bilirubin $[<$ or $>N V], C-P$ [A vs. B], LDH as a continuous variable, PLR [< or $>16$ ], ECOG [0 vs. $>0$ ], ALBI grade [1 vs. 2], portal vein thrombosis [yes vs. no], SII [ $<360$ vs. $>360$ ], and BCLC stage [B vs. C]) identified increase in LDH, age $\geq 70$ years, other etiology, ECOG $>0$, albumin $<35$, ALBI grade 2 , and AST $>40$ as prognostic factors for poorer OS based on the $5 \%$ significance level. The results of other models of multivariate analysis are shown in Table 4.

\section{Discussion}

In this retrospective study, we evaluated the OS of advanced HCC patients treated with sorafenib in real-life clinical setting. The analysis showed that age $>70$ years, performance status ECOG $>0$, HCC etiologies other than HCV or HBV infection, high levels of LDH, albumin 
$<35$, ALBI grade 2 , and AST $>40$ were associated with poorer OS. Our study demonstrates that, in real-life setting, hepatic function is the real indicator of the prognosis.

Despite the different type of analysis, the characteristics of the population of our study are consistent with the data of the SHARP trial [6]. Males were definitely more prevalent than females ( 89.4 and $10.6 \%$ vs. 87 and 13\%, respectively), median age of patients was 69 years (vs. 65 years), an ECOG Performance status of 0 was represented by $52.2 \%$ of patients (vs. $54 \%)$. A lower proportion of patients (71.2 vs. 83\%) were BCLC stage C and C-P A (88.1 vs. $97 \%$ ). Of note, median OS of 12.3 months described here is similar to that of 10.7 months reported in the above-mentioned landmark trial, thus suggesting that real-life benefit of sorafenib is similar to that reported in randomized clinical trials. The multivariate analysis showed that ALBI grade, age, and etiology were the only always positive factors.

Compared to C-P score, ALBI grade has been established by Johnson et al. [23] as a simple, evidence-based, and objective method of assessing liver function in HCC. Incorporating only serum bilirubin and albumin, it stratifies patients with HCC into three categories (with grade 3 considered the worse) and it is able to highlight distinct prognostic subgroups within C-P grade A. In our study, ALBI grade 2, rather than any grade of C-P score, was identified as a negative prognostic factor suggesting its stronger role in evaluating hepatic impairment. Our study outlines the superiority of ALBI grade over C-P score; in the univariate analysis, C-P B (vs. A) and ALBI grade 2 (vs. 1) were identified as potential prognostic factor for poorer OS, but C-P disappears when considered in multivariate analysis, whereas ALBI grade constantly remains. Although not in a real-life setting, Abdel-Rahman [24] also reported a positive effect on OS of lower ALBI.

Regarding the 2 factors comprised in the ALBI score (bilirubin and albumin), both albumin and bilirubin were identified as potential prognostic factors for poorer OS in the univariate analyses, but in the multivariate context only albumin $<35 \mathrm{~g} / \mathrm{L}$ emerged as an independent prognostic factor. Therefore, we show that albumin, rather than bilirubin, is the most relevant factor able to make ALBI grade the strongest prognostic factor in the multivariate analysis. This is partially in line with previously reported paper, such as that from Bruix et al. [25] in which not only albumin but also bilirubin was identified in the MV analysis.

Regarding age, our results show that patients with age $\geq 70$ years had an independent prognostic factor able to impact negatively on OS. This information has its relevance since, with the aging population, the treatment of HCC in elderly patients is becoming a global health issue. Sorafenib is the standard of care for patients with advanced HCC, regardless of age, following the results of SHARP and Asia Pacific (AP) trials; no specific results related to age were reported in the publications of those trials. In the univariate analysis of our study, no difference was identified between age $<70$ and $\geq 70$ years; therefore, sorafenib can be considered effective and safe also in elderly patients. When considering age in the context of a multivariate setting, older-age patients appear to have worse prognosis. Several and not available factors may contribute to explain this result; one of these may be represented by comorbidities, which were absent in our database and which may increase treatment-related toxicity. To date, only few studies reported the use of sorafenib in elderly patients. With the premise that the cutoff used to define "elderly" patients is varying across studies, the only prospective cohort study comparing treatment with sorafenib in two different age groups ( $<70$ vs. $\geq 70$ years) of cirrhotic patients with HCC reports that sorafenib is more effective in the elderly group in terms of time to progression and OS, but results are not statistically significant [26]. Several retrospective studies found similar results, in other words no difference in terms of OS [27].

Our analysis shows that etiology other than HBV/HCV infection, alcohol abuse, or NASH is an independent prognostic factor for worse OS. This information could be explained, at least for what concerns viral infections, by the fact that patients without known risk factors 
have not been subjected to HCC screening and therefore have been diagnosed with advancedstage HCC with worse liver function [28]. Moreover, again with regard to HCC viral etiology, subgroup analysis of the SHARP and AP trials seems to suggest a different efficacy of sorafenib in HBV- or HCV-positive subjects. There is no evidence of any improvement in OS attributable to sorafenib for patients positive for HBV and negative for HCV. The mechanism of action of the drug, together with the diversity in tumor microenvironment characteristics on the basis of HBV or HCV infection, could explain the different antitumor profile [29-31]. Other parameters that were found having a good correlation with the prognosis were AST, ECOG, and LDH.

AST but not ALT was an independent prognostic factor for poorer prognosis. Pathological processes that can lead to a higher proliferative state, tissue damage, and high tumor cell turnover tend to increase AST but not ALT, at least not to the same extent [32]; thus, probably explaining why in our study AST, and not ALT, negatively correlates with prognosis. In subset analyses of phase III SHARP and AP trials [33], no difference was found between ALT/AST, but we think that, in real life practice conditions, aminotransferases levels may naturally be greater and therefore their prognostic role may emerge.

ECOG $>0$ is an independent prognostic factor for poorer OS, in line with the abovementioned paper by Abdel-Rahman [24], which shows a significant survival difference based on ECOG score of 0 versus 1 and indicates that even minor differences in performance might impact the overall outlook of HCC patients undergoing treatment with sorafenib. Another paper by Samawi et al. [34] identified the independent prognostic role of ECOG PS (2-3 vs. $0-1)$.

As already described in several papers [35, 36], elevated levels of LDH are significantly associated with poor prognosis. The biological link between hypoxia, LDH levels, and the tumor-driven angiogenesis pathway through the abnormal activation of the hypoxia inducible factor- 1 is well established, and data from several analyses on different cancers seem to suggest that LDH levels may be a significant prognostic factor. Regarding LDH in HCC treated with systemic therapies, preclinical studies reported that high levels of LDH predict resistance to several tyrosine kinase inhibitors (TKIs), including sorafenib [37], through the maintenance of the glycolytic flow, and therefore the competition between ATP and TKI inhibition at the ATP enzymatic site on the protein kinase target of their activity. Recently, given the heterogeneity of studies and results and in order to produce the best estimate of effect size, Kong et al. [38] performed a meta-analysis of 10 studies to evaluate the prognostic role of $\mathrm{LDH}$, confirming that elevated LDH is a risk factor for different survival outcomes in HCC patients.

Another interesting finding from our study is the prognostic impact of previous treatments. RFA or TACE appear as potential prognostic factors for greatest OS. The positive impact of prior locoregional treatments is in line with the recently published report by AbdelRahman [24]. It can be easily imagined that, probably, previously treated patients had a biologically different (more favorable) HCC. Our study suggests that even $>3$ TACE did not negatively impact on $O S$ and did not negatively influence the possibility of receiving sorafenib; this is a remarkable information, since we would expect that a higher number of TACE can worsen patient prognosis.

Second-line treatment was associated with a highest survival. It has to be underlined that our analysis covers a period of time prior to the authorization of the use of new experimental drugs; thus, second-line treatment in our hospitals was essentially represented by metronomic capecitabine which seems to be an efficient and safe systemic therapy for patients who either did not tolerate sorafenib or progressed during sorafenib treatment $[18,19]$. Nowadays, we face a completely different therapeutic landscape since three new drugs received approval, based on phase III clinical trials, for the treatment of patients with HCC who previously received sorafenib: regorafenib (RESORCE [39]), cabozantinib (CELESTIAL [40]), and ramu- 
cirumab (REACH-2 [41]). It would be of particular interest to see how the introduction of those drugs will change the survival in a real-life experience.

We found a statistically significant correlation between all toxicity, dermatologic AEs, diarrhea, and good prognosis. This is not surprising, since several papers have highlighted this peculiar concept, both in retrospective and prospective studies [42-48]. The consistency of this association led Di Costanzo et al. [20] to even construct and validate a scoring system (OTE score), comprising HFSR, hypertension, and diarrhea, able to predict sorafenib effectiveness in sorafenib-treated HCC patients.

Several factors of impaired liver function, such as elevated levels of AFP or high C-P score, have been shown to be negative prognostic indicators in patients with HCC independent of the type of treatment; some of those factors, together with tumor-related variables (portal vein thrombosis, etc.), have been incorporated in a variety of staging and prognostic systems, such as BCLC $[34,49]$. Some of these baseline characteristics could also be identified as negative prognostic indicators for the OS on sorafenib treatment in univariate analyses of the phase III SHARP trial $[50,25]$. Consistently, AFP $>400$, C-P B, presence of portal vein thrombosis, and BCLC stage $\mathrm{C}$ were associated with poorer OS in the univariate analysis of our cohort, but their effect on OS disappeared when considered in the context of the multivariate analyses. Of note, C-P B (vs. A) was associated with shorter OS, in line with the prospective and real-life GIDEON study [8].

Finally, no correlation was found between immune inflammation indicators and prognosis in multivariate analysis. As reported in our previous paper [22], NLR was not associated with a different clinical outcome, in contrast to what was observed in the subanalysis of SHARP and AP trials conducted by Bruix et al. [25] in which high NLR had a detrimental effect on OS. This difference may be at least in part explained by the different populations in exams; prevalence of cirrhosis in our study is much higher, and this disease stage is associated with a low number of neutrophils, lymphocytes, and platelet levels.

The main strengths of our study are the large sample size and the multicenter enrollment of patients in a field-practice setting. We analyzed medical data collected under real-life conditions, therefore mimicking routine care and thus providing high external validity and high generalizability. The main weakness of our study is, on the other hand, the retrospective nature of data, which implicates collection of studies with different characteristics and endpoints, missing data, etc. To overcome, at least in part, this aspect, cases were consecutively selected, thus reducing potential bias. Another limit is represented by the fact that, given the homogeneity of the population, the applicability of the current results is restricted to non-Asian patients.

\section{Conclusion}

In advanced HCC patients, the prediction of prognosis is extremely complex because survival is affected by both tumor burden and liver function. In our study, we have provided insights into the impact of baseline characteristics on the OS of sorafenib-treated HCC patients in real-life setting, highlighting that hepatic function, patient-centered variables, and HCC etiology, rather than tumor stage, AFP, and immune inflammation indicators, have prognostic value. These findings might have implications in terms of therapeutic decision-making and patient counseling, especially now that, after 10 years of sorafenib solitude, the therapeutic scenario for advanced HCC is becoming more and more multifaceted. 


\section{Statement of Ethics}

The study protocol was reviewed and approved by the local Ethics Committee (CEIIAV: Ethics Commitee IRST IRCCS AVR). Study number IRST B041, protocol number 5482/v.1, intern. code L3P1192. All patients signed their written informed consent.

\section{Disclosure Statement}

The authors declare that they have no conflicts of interest.

\section{Funding Sources}

None.

\section{Author Contributions}

A.C.G.: conception and design of the work; analysis and interpretation of data; review, drafting, and final approval of the manuscript. G.O. and G.R.: writing the paper. All authors: acquisition, analysis, and interpretation of data; review and final approval of the manuscript.

\section{References}

1 International Agency for Research on Cancer, World Health Organization. Cancer today. Available at: https:// gco.iarc.fr/today/ home.

2 Bray F, Ferlay J, Soerjomataram I, Siegel RL, Torre LA, Jemal A. Global cancer statistics 2018: GLOBOCAN estimates of incidence and mortality worldwide for 36 cancers in 185 countries. CA Cancer J Clin. 2018 Nov;68(6): 394-424.

3 Cronin KA, Lake AJ, Scott S, Sherman RL, Noone AM, Howlader N, et al. Annual Report to the Nation on the Status of Cancer, part I: national cancer statistics. Cancer. 2018 Jul;124(13):2785-800.

4 Villanueva A. Hepatocellular Carcinoma. N Engl J Med. 2019 Apr;380(15):1450-62.

5 Bertot LC, Adams LA. Trends in hepatocellular carcinoma due to non-alcoholic fatty liver disease. Expert Rev Gastroenterol Hepatol. 2019 Feb;13(2):179-87.

6 Llovet JM, Ricci S, Mazzaferro V, Hilgard P, Gane E, Blanc JF, et al.; SHARP Investigators Study Group. Sorafenib in advanced hepatocellular carcinoma. N Engl J Med. 2008 Jul;359(4):378-90.

7 Cheng AL, Kang YK, Chen Z, Tsao CJ, Qin S, Kim JS, et al. Efficacy and safety of sorafenib in patients in the AsiaPacific region with advanced hepatocellular carcinoma: a phase III randomised, double-blind, placebocontrolled trial. Lancet Oncol. 2009 Jan;10(1):25-34.

8 Marrero JA, Kudo M, Venook AP, Ye SL, Bronowicki JP, Chen XP, et al. Observational registry of sorafenib use in clinical practice across Child-Pugh subgroups: the GIDEON study. J Hepatol. 2016 Dec;65(6):1140-7.

9 Kudo M, Finn RS, Qin S, Han KH, Ikeda K, Piscaglia F, et al. Lenvatinib versus sorafenib in first-line treatment of patients with unresectable hepatocellular carcinoma: a randomised phase 3 non-inferiority trial. Lancet. 2018 Mar;391(10126):1163-73.

10 El-Khoueiry AB, Sangro B, Yau T, Crocenzi TS, Kudo M, Hsu C, et al. Nivolumab in patients with advanced hepatocellular carcinoma (CheckMate 040): an open-label, non-comparative, phase 1/2 dose escalation and expansion trial. Lancet. 2017 Jun;389(10088):2492-502.

11 Sangro B, Park JW, Dela Cruz CM, Anderson J, Lang L, Neely J, et al. A randomized, multicenter, Phase III study of nivolumab vs Sorafenib as first-line treatment in patients (pts) with advanced hepatocellular carcinoma (HCC): CheckMate-459. J Clin Oncol. 2016;34(15 suppl):TPS4147.

12 Marisi G, Cucchetti A, Ulivi P, Canale M, Cabibbo G, Solaini L, et al. Ten years of sorafenib in hepatocellular carcinoma: are there any predictive and/or prognostic markers? World J Gastroenterol. 2018 Sep;24(36): 4152-63.

13 Casadei Gardini A, Marisi G, Faloppi L, Scarpi E, Foschi FG, Iavarone M, et al. eNOS polymorphisms and clinical outcome in advanced HCC patients receiving sorafenib: final results of the ePHAS study. Oncotarget. 2016 May;7(19):27988-99.

14 Casadei Gardini A, Scarpi E, Marisi G, Foschi FG, Donati G, Giampalma E, et al. Early onset of hypertension and serum electrolyte changes as potential predictive factors of activity in advanced HCC patients treated with sorafenib: results from a retrospective analysis of the HCC-AVR group. Oncotarget. 2016 Mar; 7(12):15243-51. 
15 Casadei Gardini A, Marisi G, Scarpi E, Scartozzi M, Faloppi L, Silvestris N, et al. Effects of metformin on clinical outcome in diabetic patients with advanced HCC receiving sorafenib. Expert Opin Pharmacother. 2015; 16(18):2719-25.

16 Casadei Gardini A, Faloppi L, De Matteis S, Foschi FG, Silvestris N, Tovoli F, et al. Metformin and insulin impact on clinical outcome in patients with advanced hepatocellular carcinoma receiving sorafenib: validation study and biological rationale. Eur J Cancer. 2017 Nov;86:106-14.

17 Casadei Gardini A, Scarpi E, Foschi FG, Marisi G, Maltoni M, Frassineti GL. Impact of physician experience and multidisciplinary team on clinical outcome in patients receiving sorafenib. Clin Res Hepatol Gastroenterol. 2018 Dec;S2210-7401(18)30262-6

18 Casadei Gardini A, Foca F, Scartozzi M, Silvestris N, Tamburini E, Faloppi L, et al. Metronomic capecitabine versus best supportive care as second-line treatment in hepatocellular carcinoma: a retrospective study. Sci Rep. 2017 Feb;7(1):42499.

19 Trevisani F, Brandi G, Garuti F, Barbera MA, Tortora R, Casadei Gardini A, et al. Metronomic capecitabine as second-line treatment for hepatocellular carcinoma after sorafenib discontinuation. J Cancer Res Clin Oncol. 2018 Feb;144(2):403-14.

20 Di Costanzo GG, Casadei Gardini A, Marisi G, Foschi FG, Scartozzi M, Granata R, et al. Validation of a Simple Scoring System to Predict Sorafenib Effectiveness in Patients with Hepatocellular Carcinoma. Target Oncol. 2017 Dec;12(6):795-803.

21 Fornari F, Pollutri D, Patrizi C, La Bella T, Marinelli S, Casadei Gardini A, et al. In Hepatocellular Carcinoma miR-221 Modulates Sorafenib Resistance through Inhibition of Caspase-3-Mediated Apoptosis. Clin Cancer Res. 2017 Jul;23(14):3953-65.

22 Casadei Gardini A, Scarpi E, Faloppi L, Scartozzi M, Silvestris N, Santini D, et al. Immune inflammation indicators and implication for immune modulation strategies in advanced hepatocellular carcinoma patients receiving sorafenib. Oncotarget. 2016 Oct;7(41):67142-9.

23 Johnson PJ, Berhane S, Kagebayashi C, Satomura S, Teng M, Reeves HL, et al. Assessment of liver function in patients with hepatocellular carcinoma: a new evidence-based approach-the ALBI grade. J Clin Oncol. 2015 Feb;33(6):550-8.

24 Abdel-Rahman 0. Impact of baseline characteristics on outcomes of advanced HCC patients treated with sorafenib: a secondary analysis of a phase III study. J Cancer Res Clin Oncol. 2018 May;144(5):901-8.

25 Bruix J, Cheng AL, Meinhardt G, Nakajima K, De Sanctis Y, Llovet J. Prognostic factors and predictors of sorafenib benefit in patients with hepatocellular carcinoma: analysis of two phase III studies. J Hepatol. 2017 Nov;67(5):999-1008.

26 Di Costanzo GG, Tortora R, De Luca M, Galeota Lanza A, Lampasi F, Tartaglione MT, et al. Impact of age on toxicity and efficacy of sorafenib-targeted therapy in cirrhotic patients with hepatocellular carcinoma. Med Oncol. 2013 Mar;30(1):446.

27 Brunot A, Le Sourd S, Pracht M, Edeline J. Hepatocellular carcinoma in elderly patients: challenges and solutions. J Hepatocell Carcinoma. 2016 Jun;3:9-18.

28 Ogasawara S, Chiba T, Ooka Y, Kanogawa N, Saito T, Motoyama T, et al. Sorafenib treatment in Child-Pugh A and B patients with advanced hepatocellular carcinoma: safety, efficacy and prognostic factors. Invest New Drugs. 2015 Jun;33(3):729-39.

29 Jackson R, Psarelli EE, Berhane S, Khan H, Johnson P. Impact of Viral Status on Survival in Patients Receiving Sorafenib for Advanced Hepatocellular Cancer: A Meta-Analysis of Randomized Phase III Trials. J Clin Oncol. 2017 Feb;35(6):622-8.

30 Casadei Gardini A, Frassineti GL, Foschi FG, Ercolani G, Ulivi P. Sorafenib and Regorafenib in HBV- or HCVpositive hepatocellular carcinoma patients: analysis of RESORCE and SHARP trials. Dig Liver Dis. 2017 Aug; 49(8):943-4.

31 Casadei Gardini A, Puzzoni M, Montagnani F, Marisi G, Tamburini E, Cucchetti A, et al. Profile of lenvatinib in the treatment of hepatocellular carcinoma: design, development, potential place in therapy and network meta-analysis of hepatitis B and hepatitis C in all Phase III trials. OncoTargets Ther. 2019 Apr;12:2981-8.

32 Bezan A, Mrsic E, Krieger D, Stojakovic T, Pummer K, Zigeuner R, et al. The Preoperative AST/ALT (De Ritis) Ratio Represents a Poor Prognostic Factor in a Cohort of Patients with Nonmetastatic Renal Cell Carcinoma. J Urol. 2015 Jul;194(1):30-5.

33 Cheng AL, Guan Z, Chen Z, Tsao CJ, Qin S, Kim JS, et al. Efficacy and safety of sorafenib in patients with advanced hepatocellular carcinoma according to baseline status: subset analyses of the phase III Sorafenib Asia-Pacific trial. Eur J Cancer. 2012 Jul;48(10):1452-65.

34 Samawi HH, Sim HW, Chan KK, Alghamdi MA, Lee-Ying RM, Knox JJ, et al.; CHORD Consortium. Prognosis of patients with hepatocellular carcinoma treated with sorafenib: a comparison of five models in a large Canadian database. Cancer Med. 2018 May;7(7):2816-25.

35 Scartozzi M, Faloppi L, Bianconi M, Giampieri R, Maccaroni E, Bittoni A, et al. The role of LDH serum levels in predicting global outcome in HCC patients undergoing TACE: implications for clinical management. PLoS One. 2012;7(3):e32653.

36 Faloppi L, Scartozzi M, Bianconi M, Svegliati Baroni G, Toniutto P, Giampieri R, et al. The role of LDH serum levels in predicting global outcome in HCC patients treated with sorafenib: implications for clinical management. BMC Cancer. 2014 Feb;14(1):110. 
37 Faloppi L, Bianconi M, Memeo R, Casadei Gardini A, Giampieri R, Bittoni A, et al. Lactate Dehydrogenase in Hepatocellular Carcinoma: Something Old, Something New. BioMed Res Int. 2016;2016:7196280.

38 Kong W, Zuo X, Liang H, Hu J, Zhang H, Wang X, et al. Prognostic Value of Lactate Dehydrogenase in Patients with Hepatocellular Carcinoma: A Meta-Analysis. BioMed Res Int. 2018 Dec;2018:1723184.

39 Bruix J, Qin S, Merle P, Granito A, Huang YH, Bodoky G, et al.; RESORCE Investigators. Regorafenib for patients with hepatocellular carcinoma who progressed on sorafenib treatment (RESORCE): a randomised, doubleblind, placebo-controlled, phase 3 trial. Lancet. 2017 Jan;389(10064):56-66.

40 Abou-Alfa GK, Meyer T, Cheng AL, El-Khoueiry AB, Rimassa L, Ryoo BY, et al. Cabozantinib in Patients with Advanced and Progressing Hepatocellular Carcinoma. N Engl J Med. 2018 Jul;379(1):54-63.

41 Zhu AX, Kang YK, Yen CJ, Finn RS, Galle PR, Llovet JM, et al.; REACH-2 study investigators. Ramucirumab after sorafenib in patients with advanced hepatocellular carcinoma and increased $\alpha$-fetoprotein concentrations (REACH-2): a randomised, double-blind, placebo-controlled, phase 3 trial. Lancet Oncol. 2019 Feb;20(2):28296.

42 Wang E, Xia D, Bai W, Wang Z, Wang Q, Liu L, et al. Hand-foot-skin reaction of grade $\geq 2$ within sixty days as the optimal clinical marker best help predict survival in sorafenib therapy for HCC. Invest New Drugs. 2019 Jun;37(3):401-414.

43 Vincenzi B, Santini D, Russo A, Addeo R, Giuliani F, Montella L, et al. Early skin toxicity as a predictive factor for tumor control in hepatocellular carcinoma patients treated with sorafenib. Oncologist. 2010;15(1):85-92.

44 Yada M, Masumoto A, Motomura K, Tajiri H, Morita Y, Suzuki H, et al. Indicators of sorafenib efficacy in patients with advanced hepatocellular carcinoma. World J Gastroenterol. 2014 Sep;20(35):12581-7.

45 Shomura M, Kagawa T, Shiraishi K, Hirose S, Arase Y, Koizumi J, et al. Skin toxicity predicts efficacy to sorafenib in patients with advanced hepatocellular carcinoma. World J Hepatol. 2014 Sep;6(9):670-6.

46 Otsuka T, Eguchi Y, Kawazoe S, Yanagita K, Ario K, Kitahara K, et al.; Saga Liver Cancer Study Group. Skin toxicities and survival in advanced hepatocellular carcinoma patients treated with sorafenib. Hepatol Res. 2012 Sep;42(9):879-86.

47 Bettinger D, Schultheiss M, Knüppel E, Thimme R, Blum HE, Spangenberg HC. Diarrhea predicts a positive response to sorafenib in patients with advanced hepatocellular carcinoma. Hepatology. 2012 Aug;56(2):789_ 90.

48 Koschny R, Gotthardt D, Koehler C, Jaeger D, Stremmel W, Ganten TM. Diarrhea is a positive outcome predictor for sorafenib treatment of advanced hepatocellular carcinoma. Oncology. 2013;84(1):6-13.

49 Wörns MA, Koch S, Niederle IM, Marquardt JU, Nguyen-Tat M, Gamstätter T, et al. The impact of patient and tumour baseline characteristics on the overall survival of patients with advanced hepatocellular carcinoma treated with sorafenib. Dig Liver Dis. 2013 May;45(5):408-13.

50 Raoul JL, Bruix J, Greten TF, Sherman M, Mazzaferro V, Hilgard P, et al. Relationship between baseline hepatic status and outcome, and effect of sorafenib on liver function: SHARP trial subanalyses. J Hepatol. 2012 May; 56(5):1080-8. 